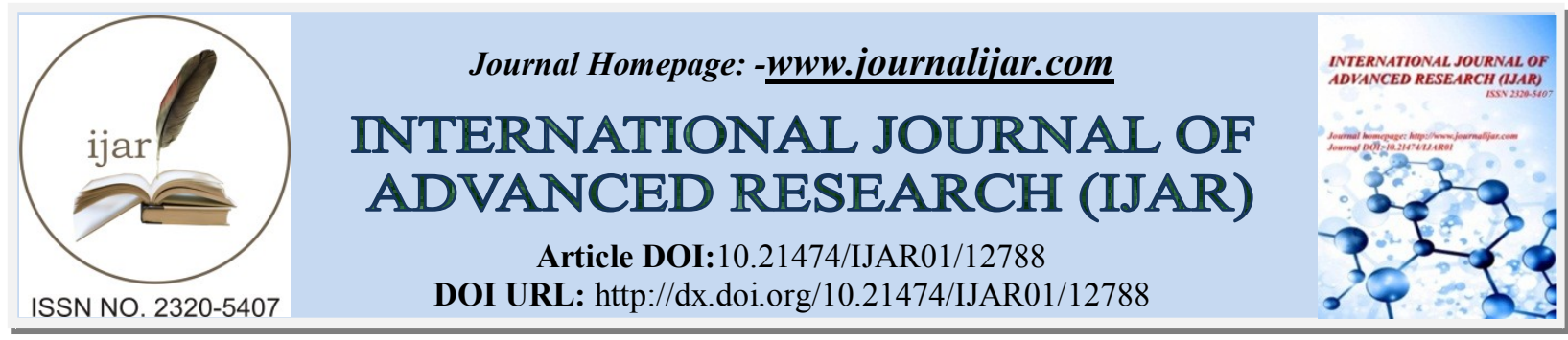

RESEARCH ARTICLE

\title{
CAUSE D'HEMORRAGIE POST PARTUM A LAQUELLE IL FAUT PENSER: PSEUDO-ANEVRISME DE L'ARTERE UTERINE APRES UNE CESARIENNE, A PROPOS D'UN CAS
}

\section{Meimouna Mohamed Lemine Issehaghe ${ }^{1}$, Khadija Bouras ${ }^{1}$, NessibaAbdelkadr Mohamed Zeine ${ }^{1}$, Nisrine Mamouni $^{1}$, Sanae Errarhay ${ }^{1}$, Chahrazed Bouchikhi ${ }^{1}$, Abdel Aziz Banani ${ }^{1}$, Farid Aassouani ${ }^{2}$ and Moustapha Maaroufi $^{2}$}

1. Service De GynecologieObstetrique I CHU Hassan II Fes, Faculté de Médecineet dePharmacie de FES, UniversitéSidi Mohamed Ben Abdallah, Maroc.

2. Service De Radioligie, CHU Hassan II de Fes.

\section{Manuscript Info}

Manuscript History

Received: 25 February 2021

Final Accepted: 30 March 2021

Published: April 2021

Key words:-

Post Partumhemorrhages, Pseudo Aneurysm Of The Uterine Artery, Color

Doppler, Embolization

\begin{abstract}
Pseudoaneurysm of the uterine artery is a rare and poorly understood complication of Caesarean section or endocavitary manipulation. It can be responsible for severe postpartum hemorrhage, most often secondary or retarded. We present here a case of pseudoaneurysm of the left uterine artery diagnosed and treated in the gynecological-obstetrics department I, CHU HASSAN II DE FES. The diagnosis was guided by ultrasound but uterine arteriography of the uterine arteries remains a determining factor in establishing and making the diagnosis and also guiding the treatment.Selective arterial embolization was the treatment of choice. It has excluded the lesion, but vascular ligature or hysterectomy may be necessary if the patient is hemodynamically unstable.
\end{abstract}

Copy Right, IJAR, 2021,. All rights reserved.

\section{Introduction:-}

Le pseudo anévrisme de l'artèreutérine se formelorsquel'artèreestléséeoublessée. Tout en maintenant le contact avec le vaisseaud'origine, le sang extravasé se dissèque à travers les tissus.

le pseudo- anévrysme de l'artèreutérine(PAU) estune cause importanted'hémorragie de post-partum retardée. Ilspeuventsurvenir suit à des accouchements par voiebasseou par césarienne, ou suit d'un curetage, des myomectomies ouconisationcervicale. Le mécanismephysiopathologique de leursurvenueest le plus souventtraumatique(Parr K, et all; 2018).

\section{Observation:-}

Unepatienteâgée de 30 ans, troisièmegeste, troisièmepare, sans antécédentspathologiquesparticuliers, a présenté 10 jours après un accouchement par césarienne, troisepisodedesmétrorragies de moyenneabondence. L'examenclinique a révéléunepatientepâle, hypotendue $(80 / 40 \mathrm{mmHg})$, tachycarde $(120$ battements par minute).L'examengynécologique a montré des saignements des moyennesabondances en provenant de l'endocol.Le bilanbiologique a mis en évidenceuneanémie avec unehémoglobinémie à $6 \mathrm{~g} / \mathrm{dL}$.Le reste du bilanbiologiquen'a pas révéléd'anomalie en particulier de l'hémostase. Après stabilisationhémodynamique, uneéchographiepelvienne a étéréaliséeet a objectivéune formation anechogenejuxta-utérine gauche, vascularisée au Doppler couleur, comportant 
un flux artériel turbulent (Fig. 1). Le diagnostic d'unelésionanévrismaleutérine gauche aétéfortementsuspecté. Unangioscannerabdominopelvien a confirmé la présence d'un pseudo-anévrisme de la portion cervico-vaginale de l'artèreutérine gauche, sans extravasation du produit de contraste, niépanchementpéritonéal (Fig. 2).Uneartériographie à visée diagnostic et thérapeutique a étérealisée. L'examenaétéréalisé par ponction de l'artèrefémorale gauche et cathétérisme des artèreshypogastriques, puis du tronc de l'artèreutérine gauche (Fig. 3.A). L'opacification de l'artèrehypogastrique gauche individualise un pseudo anévrysmesacculaire, sur le trajet ascendant de l'artèreutérine gauche (Fig. 3.B).Puisun micro cathétérisme de l'artèreutérine gauche et embolisation de sabranche ascendant y compris le pseudo anévrysme par un mélange de col biologique et de Lipiodol (Fig. 4.A). Le contrôle final a montrél'exclusion du sac anévrismal (Fig. 4.B).L'évolutionaétémarquéeparl'arrêt des métrorragies.

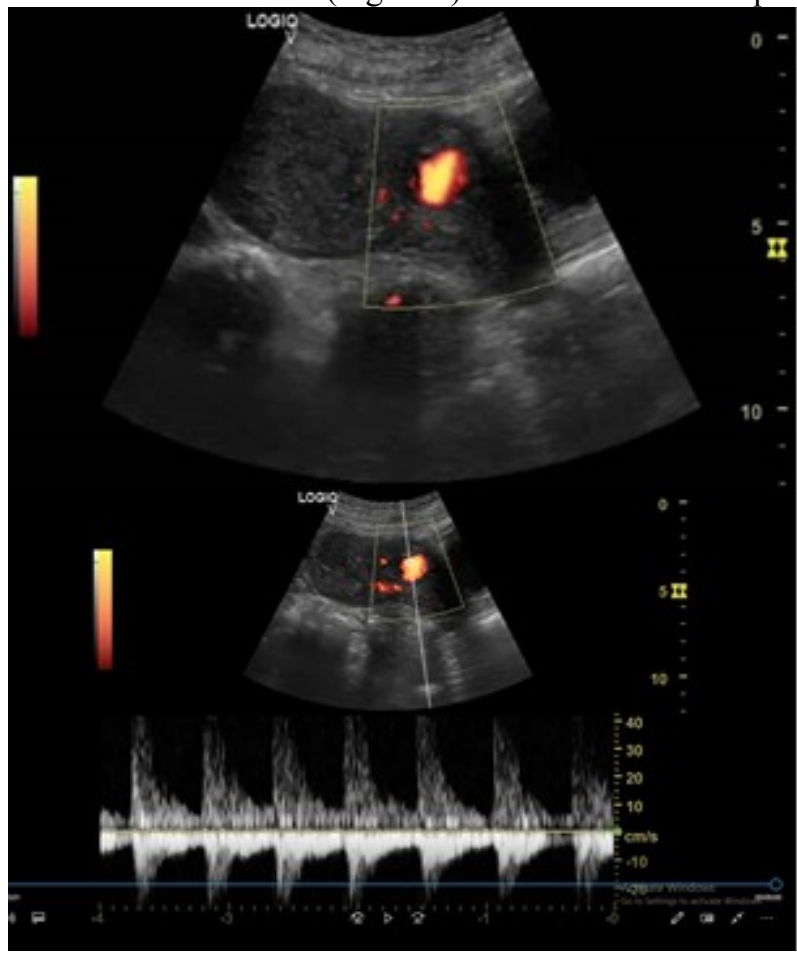

Figure 1:-Échographie-Doppler pelvienneendovaginale : formation juxta-utérine gauche, anéchogène, richementvascularisée au Doppler couleur, avec un flux artérielturbulant et un phénomèned'aliasings.

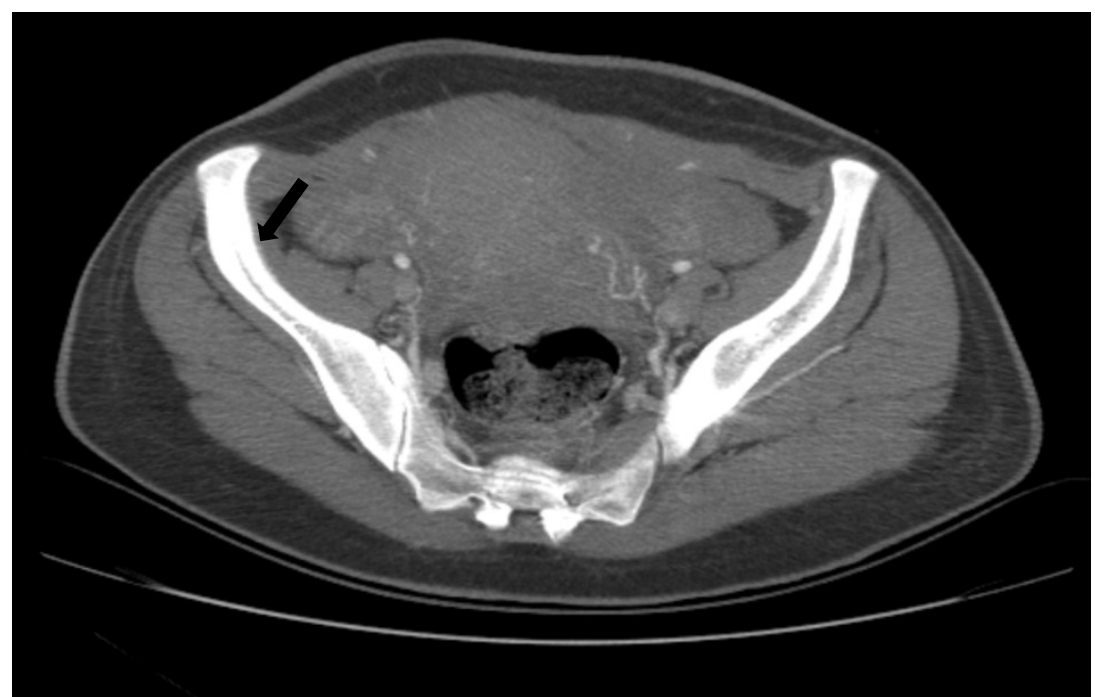

Figure 2 (A) :- TDM abdominaleinjectée ( temps artériel ) : Individualisationd'une petite image d'addition, sacciforme, au dépendd'unebranche de l'artèreutérine gauche (flèche noire ); en rapport avec un pseudoanévrysme.A 


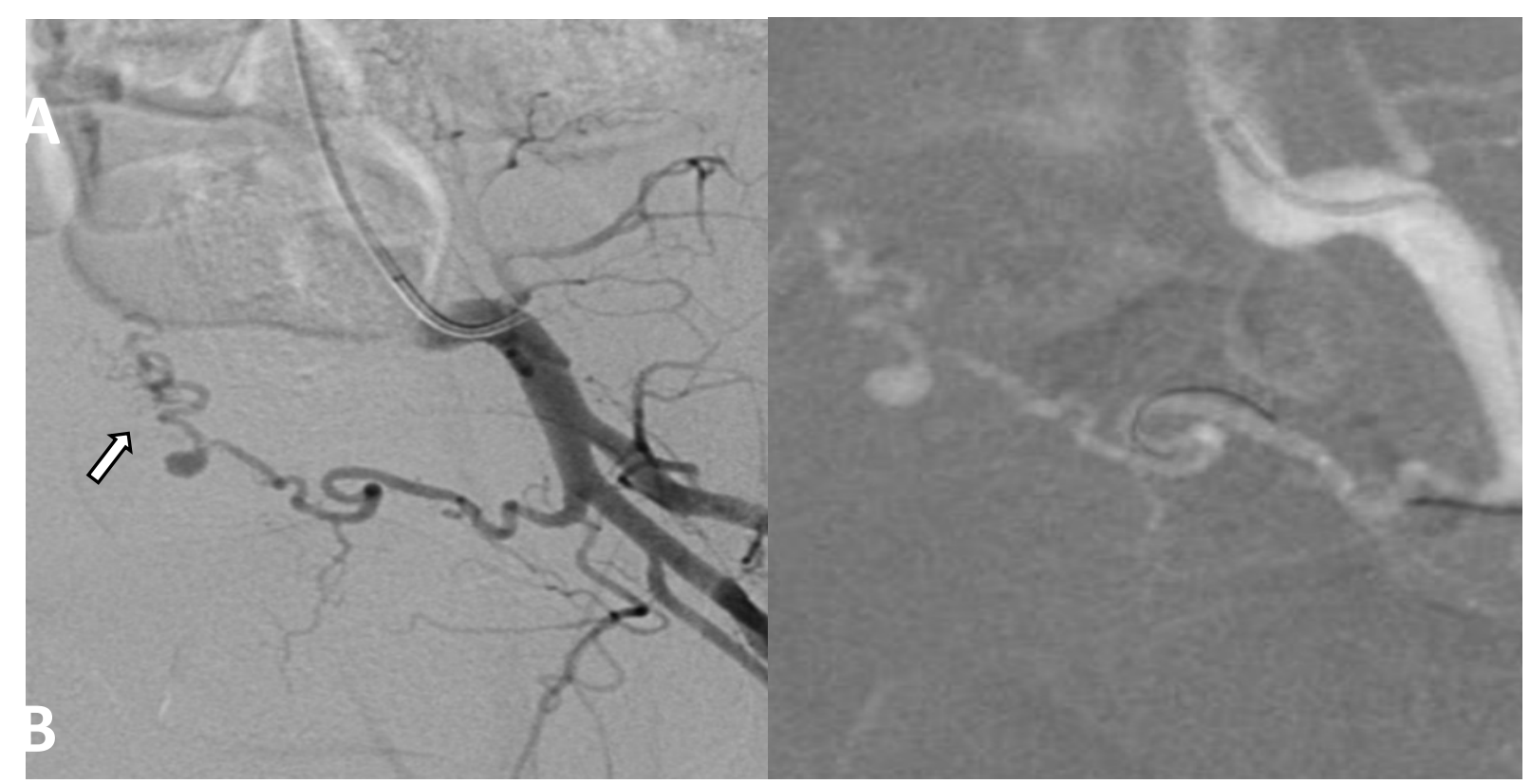

Figure 3:-Artériographie :L'opacification de l'artèrehypogastrique gauche individualise un pseudo anévrysmesacculaire, sur le trajet ascendant de l'artèreutérine gauche.

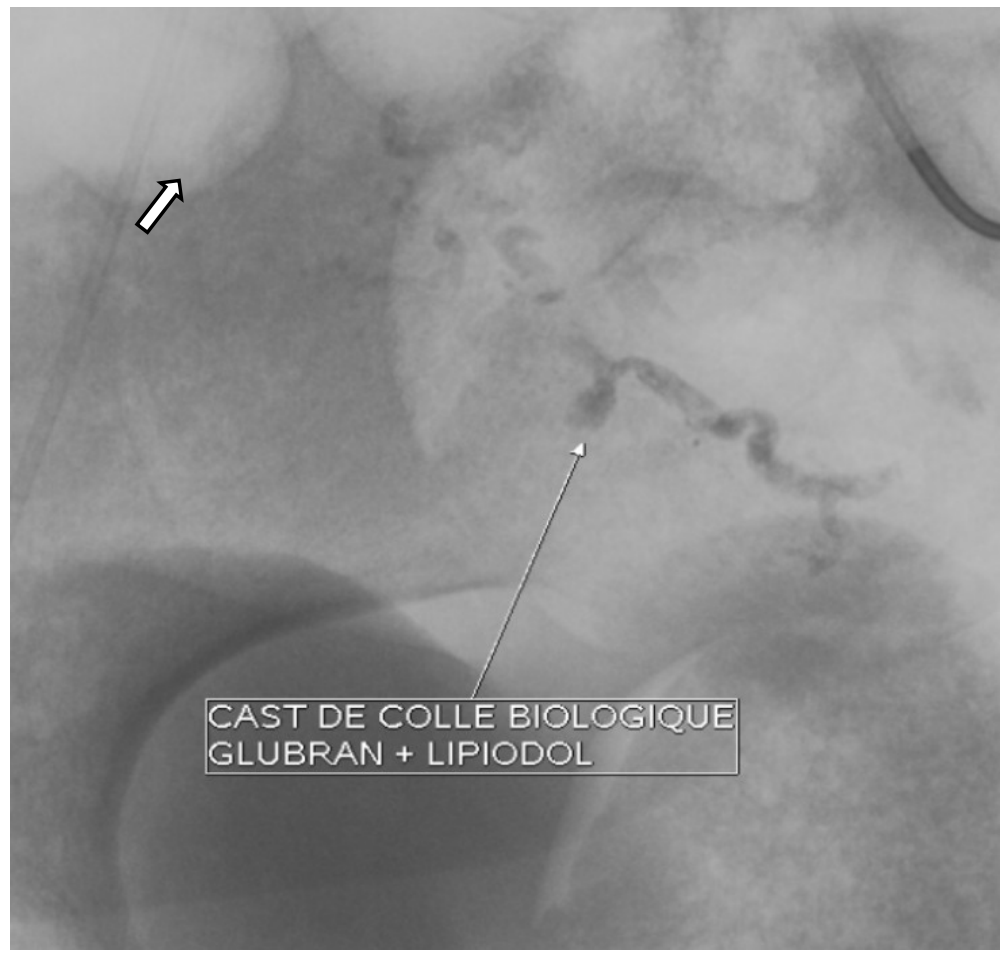

Figure 4 (A) Artériographie :

Micro cathérisme de l'artèreutérine gauche etembolisation de sabrancheascendante y compris le pseudo anévrysme par un mélange de col biologique et de Lipiodol. 


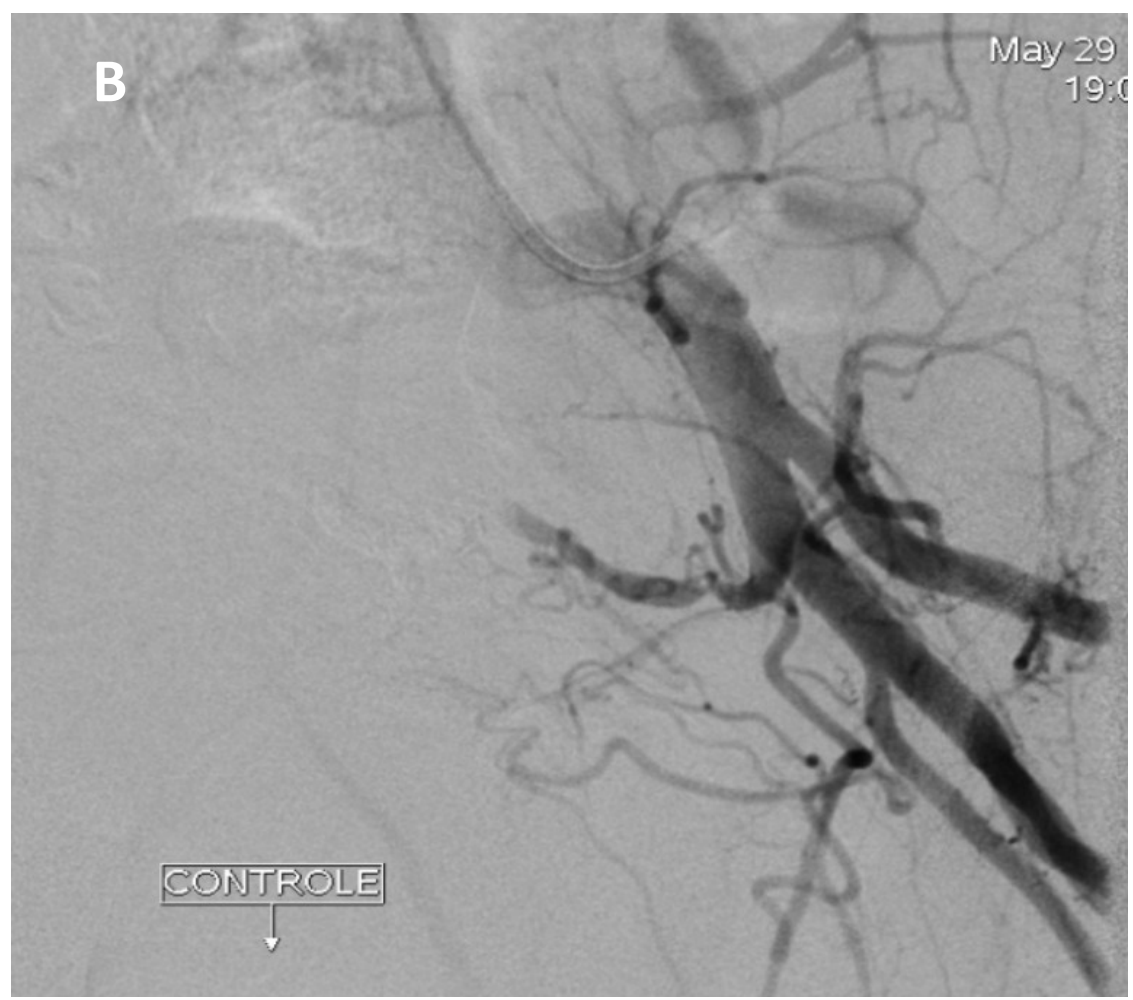

Figure 4(B):- Artériographie :Contrôlesatisfaisant.

\section{Discussion:-}

L'hémorragie du post-partum (HPP), estl'un des principales causes de mortalitématernelle, ellepeutsurvenirdans les $24 \mathrm{~h}$ suivantl'accouchement (HPP primaire) ou pendant la périodeallant de $24 \mathrm{~h}$ après l'accouchement à la sixièmesemaine du post partum (HPP) secondaireou tardive. Les cas de PAU rapportésdans la littératuresont le plus souventsurvenuesdans un contextetraumatiqueslors d'un accouchement par voiebasseou par césarienneoulorsd'une 'interruption de grossesse, unedélivranceartificielle, une dilatation cervicaleoulors d'un curetage(Zhang N, et all ; 2017)

La prévalenceestdifficile à déterminer en raison de rareté de pseudo-anévrysme de l'artèreutérine. Uneprévalence de 2 à 3/1000 accouchements ontétérapportésmaisdanscetteétude les parturientesasymptomatiquesontétéinclus(Parr K, et all; 2018).

La PAU se présentegénéralementcommeunehémorragie du post-partum tardive ousecondaire, dans la littérature,unesériede 18 cas a étépublié avec unehémorragie de survenustardivesdans $66,7 \%$ des cas avec un intervallemoyen entre l'accouchement et l'hémorragique de 28jours. Le tableau cliniquepeutvarier des saignementsinsidieuxetminime à unehémorragie massive. Le plus Souvent, hémorragieestattribuée à des causes plus fréquentesd'hémorragie du post-partum, tellesqu'unerétentionplacentaire, atonieutérineouuneendométrite postpartum. Le manque de reconnaissance de la PAU peutconduire à une retard ou absence de prise en charge adapté, avec parfois le recours à l'hystérectomied'hémostasedans les casd'hémorragiegrave(Zhang N, et all ; 2017)

Le mécanisme PA estlié à la transfixion ou à la lacération des paroisartérielleslors de traumatismesutérins. À la différence des " vrais » anévrismes, constitués des trois couches pariétales (intima, média, adventice), les pseudoanévrismessontconstituésd'une simple couchefibreuse formant unepochedanslaquellecirculeun flux turbulent. En raison de la haute pressiondansl'artère, le sang peutsortir à travers le tissuconjonctif, entraînant des saignementsvaginaux. Cetissuconjonctifpeut se romprefacilement, entraînantunehémorragieimportantedans la cavitéutérine(Eason DE, et all; 2006).

Le pseudo-anévrismepeutégalement se refermer, conduisant à des épisodes de saignement vaginal plutôtqu'unépisodeaigue(Kuwata T, et all; 2009)commeilétait le cas de notrepatiente. La modalitédiagnostique de 
choixestl'échographieendovaginalecouplée au Doppler couleur. PAUs se présententgénéralementcommeune masse hypoéchogène avec un le signe de «yin-yang» sur Doppler, reflétant le flux sanguin turbulent dans le pseudoanévrisme pendant la systole et le sang sort de la cavité pendant la diastole (Parr K, et all; 2018), et qui estresponsableparfoisd'aliasing(Keeling AN, et all; 2009), pour notrepatientel'échographieavaitmontréune image juxtautérine gauche, vascularisé au dopplercouleur, et avec aussil'aliasing.

L'angiographierestel'examenclé pour établir le diagnostic. Cette technique permet de préciserl'axeartérielnourricier, de dépister les variations anatomiques(Delesalle C, et all ; 2015)permettantaussi de guider l'embolisation. Le traitement de choix des pseudo-anévrismesutérinc'estl'embolisation, afind'éviter les risques de complications ischémiquesutérinesouovariennes(Pelage JP, et all; 2004). Pour notrepatienteelle a bénéficiéd'uneembolisationsélective de pseudo anévrysme gauche avec uncontrôlemontrantl'élimination de celui-ci.

Pour les patientes hémodynamiquement instables, ligature peropératoire des vaisseauxutérins et l'hystérectomiesont les options(Chummun K, et all; 2015)

La dilatation et le curetagesont à éviter chez cespatientes car ilpeutperturber le pseudo-anévrysme, et entraînent des hémorragies plus abondants (Yi SW et all; 2012), car l'hémorragie du post-partum retardéestsouventattribuée à unerétention des produit de conceptions, avant de procéder à toute intervention, la présence d'un PAU doitêtre recherché et éliminé.

Unefoisl'embolisationeffectuée, Les patientedoitêtresuivie au Doppler couleurimmédiatement après l'embolisation, puis à intervalles de troismois pendant un an et enfinchaqueannée pendant troisans pour confirmer la résolutioncomplète du pseudo-anévrisme(Sharma N, all; 2013), notremalade a bénéficié d'un suivieéchographiquemensuelobjectivant la disparition du PAU au troisièmemois post embolisation.

\section{Conclusion:-}

Notre observation soulèvedavantagel'importance de prise en conscience de la survenue d'un PAU rompue Par le clinicien, surtoutlors de survenue des hémorragies du post partumsecondaire avec accouchement par césarienne, maisaussiilfaut y pensermêmesil'accouchements'estdérouler sans évènementtraumatique, l'échographie fait suspecter le diagnostic dans la plus part du cas, confirmé par artériographie en permettantaussi de guider la prise en charge thérapeutique après stabilisation de la patiente par embolisation de l'artèreutérineconcerné.

\section{Références:-}

1. Chummun K, Kroon N, Flannelly G, Brophy D. Severe postcoital bleeding from a uterine artery pseudoaneurysm 4 months after, cesarean delivery. Obstet Gynecol. 2015;126(3):638-641. doi:10.1097/ AOG.0000000000000849.

2. Delesalle C, Dolley P, Beucher G, Dreyfus M, Benoist G. Lepseudoanévrysme de l'artèreutérine : une cause parfoisigno-réed'hémorragiesecondaire du post-partum. J GynecolObstetBiolReprod 2015;44:88 — 92.

3. Eason DE, Tank RA. Avoidable morbidity in a patient with pseudoaneurysm of the uterine artery after cesarean section. J Clin Ultrasound. 2006;34(8):407-411. doi:10.1002/jcu.20259.

4. Keeling AN, McGrath FP, Lee MJ. Interventional radiology inthe diagnosis, management and follow up of pseudoanevrysms.CardiovascInterventRadiol 2009;32:2 - 18.

5. Kuwata T, Matsubara S, Kaneko Y, Izumi A, Nakata M, Suzuki M. Asymptomatic uterine artery pseudoaneurysm after cesarean section. J ObstetGynaecol Res. 2010;36(2):405-410. doi:10.1111/j.1447-0756. 2009.01134.X.

6. Parr K, Hadimohd A, Browning A, Moss J. Diagnosing and treating postpartum uterine artery pseudoaneurysm. Proc (BaylUniv Med Cent). 2018 Feb 1;31(1):56-58. doi: 10.1080/08998280.2017.1400301. PMID: 29686554; PMCID: PMC5903530.

7. Pelage JP, Laissy JP. Management of life-threatening post-partum hemorrhage: indications andtechnique of arterialembolization. J GynecolObstetBiolReprod 2004;33:93-114.

8. Sharma N, Ganesh D, Devi L, Srinivasan J, Ranga U. Prompt diagnosis and treatment of uterine arcuate artery pseudoaneurysm: a case report and review of literature. J ClinDiagn Res. 2013 Oct;7(10):2303-6. doi: 10.7860/JCDR/2013/6063.3506. Epub 2013 Oct 5. PMID: 24298511; PMCID: PMC3843416. 
9. Yi SW, Lee JH. Uterine pseudoaneurysm leakage may cause delayed postpartum haemorrhage: multidetector CT with angiography and transcatheter uterine arterial embolisation. J ObstetGynaecol. 2012;32 (6):552-555. doi:10.3109/01443615.2012.694509.

10. Zhang N, Lou WH, Zhang XB, Lin JH, Di W. Antepartumhemorrhagefromprevious-cesarean-sectioneduterus as a potentialsign of uterinearterypseudoaneurysm. J Zhejiang UnivSci B. 2017 May;18(5):441-444. doi: 10.1631/jzus.B1600528. PMID: 28471117; PMCID: PMC5442977. 